\title{
Chaotic vortical flows and their manifestations
}

\author{
M. Baznat ${ }^{1, a}$, K. Gudima ${ }^{1, b}$, A. Sorin ${ }^{1, c, d}$, and O. Teryaev ${ }^{1, e}$ \\ ${ }^{1}$ Joint Institute for Nuclear Research, 141980 Dubna, Russia
}

\begin{abstract}
We study vorticity and hydrodynamic helicity in semi-peripheral heavy-ion collisions using the kinetic model of Quark-Gluon Strings. The angular momentum, which is a source of P-odd observables, is preserved with a good accuracy. We observe formation of the specific toroidal structures of the vorticity field. Their existence, accompanied by the strange chemical potential, is mirrored in the polarization of hyperons of the percent order.
\end{abstract}

\section{Introduction}

The local violation [1] of discrete symmetries in strongly interacting QCD matter is now under intensive theoretical and experimental investigations. The Chiral Magnetic Effect (CME) uses the (C)Pviolating (electro)magnetic field emerging in heavy ion collisions in order to probe the (C)P-odd effects in QCD matter.

There is an even more interesting counterpart of this effect, Chiral Vortical Effect (CVE)[2] due to coupling to P-odd medium vorticity leading to the induced electromagnetic and [3] all conservedcharge currents, in particular the baryonic ones.

The zeroth component of axial current and correspondent axial charge are related to hydrodynamical helicity

$$
H \equiv \int d V(\boldsymbol{v} \cdot \boldsymbol{w})
$$

being the projection of velocity $v$ to vorticity $w=$ curlv. This quantity is manifesting the recently discovered [4] and confirmed [5] phenomenon of separation, i.e. mirror behavior (same magnitude but different sign) in the half-spaces separated by the reaction plane

Another important P-odd observable is the baryon polarizatiion. The mechanisms analogous to CVE (known as axial vortical effect, see [6] and Ref. therein) leads to induced axial current of strange quarks which may be converted to polarization of $\Lambda$-hyperons [3, 4, 7]. Another mechanism of this polarization is provided [8] by so-called thermal vorticity in the hydrodynamical approach.

The noncentral heavy ion collisions could naturally generate a rotation (global or local, both related to vorticity) with an angular velocity normal to the reaction plane, which is their generic qualitative feature. It is naturally to expect that angular momentum conservation plays an essential role in

\footnotetext{
ae-mail: baznat@theor.jinr.ru

be-mail: gudima@cc.acad.md

$\mathrm{c}_{\text {speaker }}$

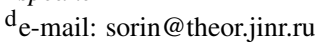

e e-mail: teryaev@theor.jinr.ru
} 
the defining the quantitative properties of vortical effects. At the same time, it remains to be studied to which extent the particles carrying the main part of angular momentum participate in the collisions.

We address these problem by performing the extensive numerical simulations $[4,9]$. We explore the distribution of angular momentum and find that the role of participant nucleons is relatively small, albeit noticeable. We study in some detail the structure of vorticity field and compare different approaches to polarization calculation. We found the peculiar toroidal "tire-like" structure manifesting themselves in the polarization of hyperons. Very different approaches to polarization calculation lead to surprisingly similar results.

\section{Modelling velocity, vorticity and helicity in kinetic model}

One of the first models designed to describe the dynamics of energetic heavy-ion collisions was the intra-nuclear cascade model developed in Dubna [10] which is based on the Monte-Carlo solution of a set of the Boltzmann-Uehling-Uhlenbeck relativistic kinetic equations with the collision terms, including cascade-cascade interactions. For particle energies below $1 \mathrm{GeV}$ it is sufficient to consider only nucleons, pions and deltas. The model includes a proper description of pion and baryon dynamics for particle production and absorption processes. In the original version the nuclear potential is treated dynamically, i.e., for the initial state it is determined using the Thomas-Fermi approximation, but later on its depth is changed according to the number of knocked-out nucleons. This allows one to account for nuclear binding. The Pauli principle is implemented by introducing a Fermi distribution of nucleon momenta as well as a Pauli blocking factors for scattered nucleons.

At energies higher than about $10 \mathrm{GeV}$, the Quark-Gluon String Model (QGSM) is used to describe elementary hadron collisions $[11,12]$. This model is based on the $1 / \mathrm{N}_{c}$ expansion of the amplitude for binary processes where $\mathrm{N}_{c}$ is the number of quark colours. Different terms of the $1 / \mathrm{N}_{c}$ expansion correspond to different diagrams which are classified according to their topological properties. Every diagram defines how many strings are created in a hadronic collision and which quark-antiquark or quark-diquark pairs form these strings. The relative contributions of different diagrams can be estimated within Regge theory, and all QGSM parameters for hadron-hadron collisions were fixed from the analysis of experimental data. The break-up of strings via creation of quark-antiquark and diquark-antidiquark pairs is described by the Field-Feynman method [13], using phenomenological functions for the fragmentation of quarks, antiquarks and diquarks into hadrons. The modified nonMarkovian relativistic kinetic equation, having a structure close to the Boltzmann-Uehling-Uhlenbeck kinetic equation, but accounting for the finite formation time of newly created hadrons, is used for simulations of relativistic nuclear collisions. One should note that QGSM considers the two lowest $\mathrm{SU}(3)$ multiplets in mesonic, baryonic and antibaryonic sectors, so interactions between almost 70 hadron species are treated on the same footing. This is a great advantage of this approach which is important for the proper evaluation of the hadron abundances and characteristics of the excited residual nuclei. The energy extremes were bridged by the QGSM extension downward in the beam energy [14].

For investigation of dynamical formation of velocity $\boldsymbol{v}$ and vorticity $\omega$ (三 rot $\boldsymbol{v}$ ) fields in relativistic heavy ion collision the coordinate space was divided into $50 \times 50 \times 100$ cells of volume $d x d y d z$ with $d x=d y=0.6 \mathrm{fm}, d z=0.6 / \gamma \mathrm{fm}$, where $\gamma$ is the gamma factor of equal velocity system of collision. In this reference system the total momentum and total energy of the produced particles were calculated in all cells for each of fixed 25 moments of time $t$ covering the interval of $10 \mathrm{fm} / \mathrm{c}$.

The results were averaged for about 10000 heavy ion collisions with identical initial conditions. The spectator nucleons of projectile or target ions, which at given time momentum do not undergone any individual collision, were included in evaluation of velocity. The velocity field in the given cell 
was defined by the following double sum over the particles in the cell and over the all simulated collisions:

$$
\boldsymbol{v}(x, y, z, t)=\frac{\sum_{i} \sum_{j} \boldsymbol{P}_{i j}}{\sum_{i} \sum_{j} E_{i j}}
$$

where $\boldsymbol{P}_{i j}$ and $E_{i j}$ are the momentum and energy of particle $i$ in the collision $j$, respectively. The vorticity was calculated using the discrete partial derivatives.

We paid a special attention to the pseudoscalar characteristics of the vorticity, that is the mentioned hydrodynamical helicity $H \equiv \int d V(\boldsymbol{v} \cdot \boldsymbol{w})$ which is related to a number of interesting phenomena in hydrodynamics and plasma physics, such as the turbulent dynamo (providing possibly additional mechanism of magnetic filed generation on the later stages of heavy-ion collisions) and Lagrangian chaos. It might be compared to the analog of topological charge $Q=\int d^{3} x J^{0}(x)$ where the current $J^{\mu}=\epsilon^{\mu \nu \rho \gamma} u_{\nu} \partial_{\rho} u_{\gamma}$ (as usual, the four-velocity $u_{v} \equiv \gamma(1, \boldsymbol{v})$ ) contributes to the hydrodynamical anomaly [15] and the polarization of hyperons [3,7]. The calculation of the topological charge which is the correct relativistic generalization of the hydrodynamical helicity leads to the extra factor $\gamma^{2}$ in the integrand. Still as the helicity itself is a more traditional quantity, we use it for the numerical calculations.

\section{Angular momentum conservation in the kinetic model}

The natural source of the P-odd observables in heavy-ion collisions is the pseudovector of angular momentum. The question immediately emerges whether it is conserved in the course of evolution governed by QGSM discussed in the previous section.. To check this we calculated the angular momentum at various moments of collision taking into account both the contributions of participants and spectators. The general structure of velocity field follows the "small bang" pattern qualitatively discussed earlier and which may be quantified by the following (see fig. 1) velocity dependence allowing to extract the "little Hubble" constant.

The averaged (over mentioned 10000 events) time dependencies of angular momenta in $\mathrm{Au}+\mathrm{Au}$ collisions at $\sqrt{s_{N N}}=5 \mathrm{Gev}$ with the impact parameter $8 \mathrm{fm}$ equal to the (transverse) radius of the nuclei are presented at figure (2). The fireball angular momentum and its ratio to the total angular momentum (appeared to be conserved in the applied model) are shown. Also, the behaviour of the absolute value of helicity changing in the accordance to angular momentum is shown. We found that the participants carry about $20 \%$ of angular momentum and that the total angular momentum of participants and spectators is conserved with a rather good accuracy and that helicity is changing in accordance with participants angular momentum (up to $t \sim 10 \mathrm{fm} / \mathrm{c}$ and decreasing afterwards) so that at least part of angular momentum is pumped into vorticity.

\section{Large-scale structures of vorticity fields}

We study the qualitative structure of velocity and vorticity fields. Our observation is that while velocity field represent the "small bang" picture, vorticity field form the relative thin toroidal "tire-like" structures. They emerge for the particular orientation of reaction plane and survive after the average over various orientations

\section{Hyperon polarization}

We compare the two rather distinct methods of determining the hyperon polarization. The first corresponds to earlier suggested[3] and explored [4] relation to induced axial current while the second one follows the procedure $[8,16]$ based on thermal vorticity. 


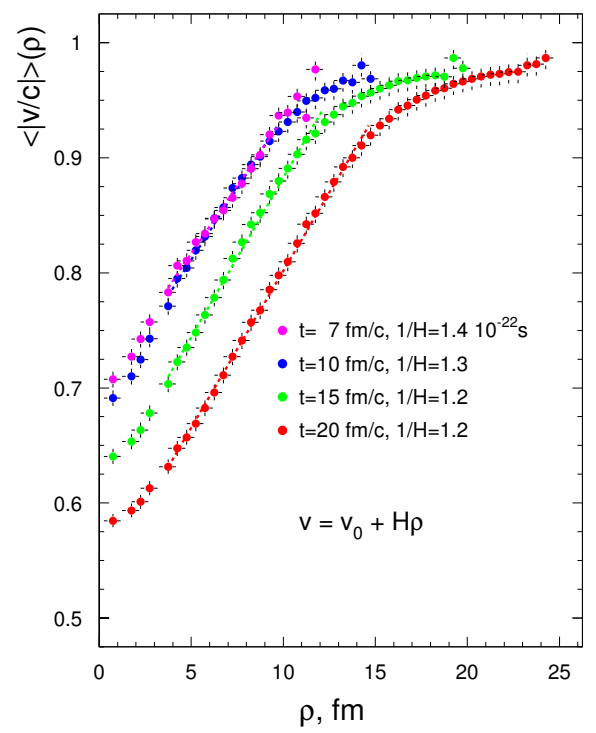

Figure 1: "Little Hubble" law

\subsection{Helicity and polarization of hyperons}

The polarization of hyperons is based on the discussed effect of helicity separation which is confirmed by the calculation in the hybrid UrQMD model manifests very similar behavior [17]. This is not surprising as the helicity is in fact generated at the hydrodynamical stage of the model, while the transition from kinetic to hydrodynamical stage should be performed similarly to our Eq. (1).

This effect might be qualitatively explained, if the perpendicular components of velocities (which are selected to have different signs) and the corresponding vorticities (assumed to have the same signs) provide the dominant contribution to the scalar product in the helicity definition. However, the numerical analysis [4] showed that the longitudinal components along the beam directions (z-axis) provide even larger contribution to the helicity than contributions from the transverse direction ( $y-$ axis). Note that comparable values of $\mathrm{z}$ and $\mathrm{y}$ components to helicity is due to larger $z$-components of velocity and $y$ - components of vorticity

So, such qualitative picture is oversimplified, but still provides a correct sign convention for the helicity-separation effect.

The energy dependence of helicity separation effect appears to be very weak, with the maximal value achieved around the NICA energy range.

The hydrodynamical helicity should give rise to the polarization of $\Lambda$-hyperons with the sign differing for the particles with "up" and "down" $y$-components of their momenta, so that the hyperons acquire the helicity in the course of their motion transverse to the reaction plane. As we already 


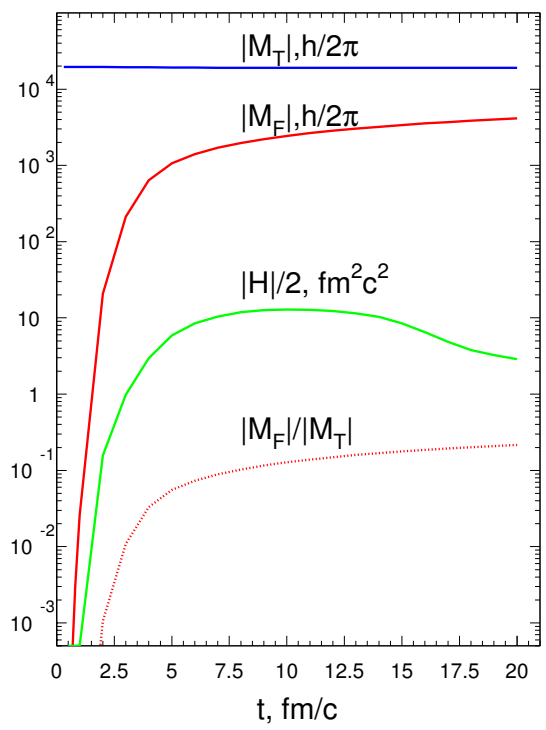

Figure 2: Angular momentum and helicity evolution

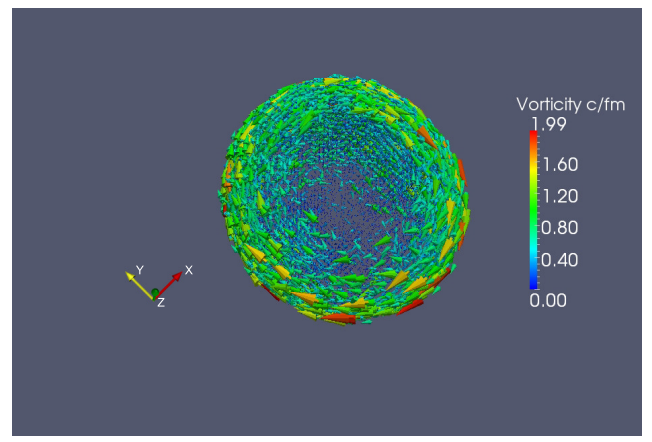

(a)

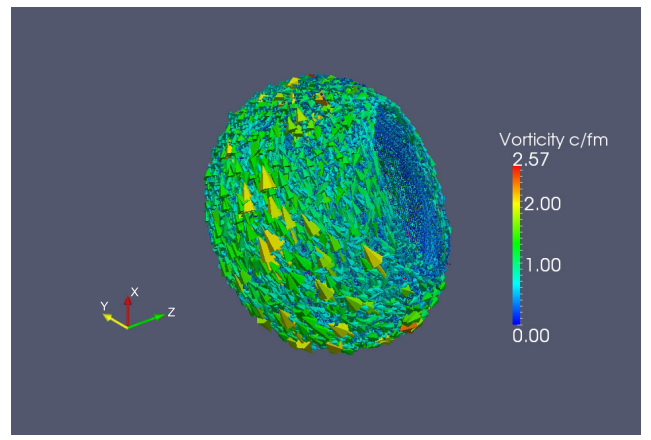

(b)

Figure 3: (a) Vorticity field for the fixed orientation of reaction plane (b) Vorticity field for the orientation of reaction plane averaged in the full $2 \pi$ region 
suggested earlier [3], the effect is pronounced at moderate (NICA) energies due to large (strange) chemical potential. the current investigation shows that, luckily, the helicity at these energies is also noticeable.

For semi-quantitative estimate of this effect one may use the average strange chiral charge produced by the zeroth component of the respective current

$$
Q_{5}^{s}=\frac{N_{c}}{2 \pi^{2}} \int d^{3} x \mu^{2}(x) \epsilon^{i j k} u_{i} \partial_{j} u_{k}=\frac{<\mu^{2}>N_{c} H}{2 \pi^{2}},
$$

where we use the mean value theorem to extract the value of the square of strange chemical potential at some point inside the integration region and get the helicity from the remaining integral. Assuming that the strange chirality is carried by the $\Lambda$ hyperons whose average number in each evemt is $\left\langle N_{\Lambda}\right\rangle$ one get the estimate for its average polarization as

$$
<P_{\Lambda}>\sim \frac{<\mu^{2}>N_{c} H}{2 \pi^{2}<N_{\Lambda}>}
$$

For numerical estimate at NICA energies, we take (see Fig. 3) $H=30 \mathrm{fm}^{2}(c=1)$ and, as a typical values, $<\mu^{2}>=900 \mathrm{MeV}^{2},<N_{\Lambda}>=15$ to get $\left\langle P_{\Lambda}>\sim 0.8 \%\right.$. This value is not large, but does not exclude the opportunity to measure the effect. Note that it is indirectly supporting the actual calculations of helicity as the obtained expression respects the density matrix positivity [20] limit $P_{\Lambda} \leq 1$. Should the helicity be much larger (resulting in the appearance of much larger value of dimensionless ratio), much larger number of hyperons production (and/or $K^{*}$-mesons) would be required to preserve the density matrix positivity. This is an example of the situation when the spin-dependent effects may be used [20] to bound the spin-averaged cross-sections from below.

Let us pass to more detailed calculations of polarization taking into account the spatial distribution of chemical potential and the kinematics of medium and produced hyperons.

$$
Q_{5}^{s}=\frac{N_{c}}{2 \pi^{2}} \int d^{3} x \mu^{2}(x) \gamma^{2} \epsilon^{i j k} u_{i} \partial_{j} u_{k}
$$

In [4] we explored the mean-value theorem, while here the spatial variation of strange chemical potential $\mu$ is taken into account. To do so, the description of kinetic distribution functions by the correspondent equilibrium equation was performed, providing the matching of kinetic and thermodynamical descriptions. As a result, the time dependence of the distribution of strange chemical potential takes the form represented at Fig. 4 The average polarization can be estimated by dividing $Q_{5}^{s}$ (2) by the number of $\Lambda$ 's, assuming that the pseudovector of axial current is proportional to the pseudovector of polarization, $Q_{5}^{s} \sim<\Pi_{0}^{\Lambda, l a b}>$. Selecting the axial charge related to the particles in the definite rapidity or transverse momentum interval, the respective dependence of polarization may be also obtained.

As the axial charge should be related to the zeroth component of hyperon polarization in laboratory frame $\Pi_{0}^{l a b}$, the transformation to hyperon rest frame should be performed. Taking into account that polarization pseudovector should be directed along $y$ axis (as it has to be collinear to $\boldsymbol{M}$ pseudovector), one get

$$
\Pi_{0}^{\Lambda, l a b}=\frac{\Pi_{0}^{\Lambda} p_{y}}{M_{\Lambda}}
$$

so that the rest frame polarization can be obtained as

$$
<\Pi_{0}^{\Lambda}>=Q_{5}^{s}<\frac{M_{\Lambda}}{N_{\Lambda} p_{y}}>
$$




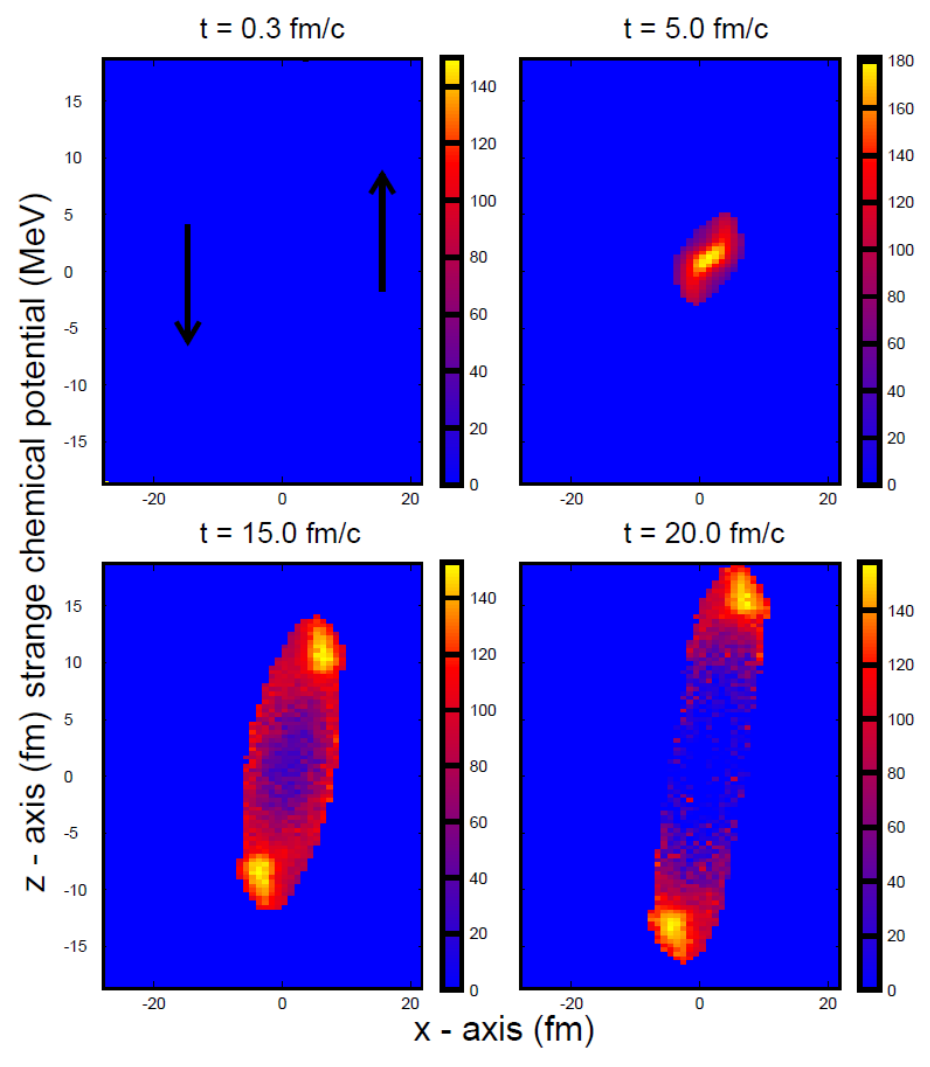

Figure 4: The time dependence of strange chemical potential.

The possible violation of positivity $\left(\left|\Pi_{\Lambda}\right| \geq 1\right)$ or even the divergence is due to the fact, that hyperons with zero $y$ component of the momentum should not have the zeroth component of polarization and therefore should not contribute to $Q_{5}^{s}$. To avoid this problem one may instead attribute the factor $p_{y} / M$ to each hyperon in the denominator of (4). Nevertheless the comparison of various approaches shows the similar scale and rapidity dependence of polarization.

\subsection{Polarization and thermal vorticity}

Another approach to polarization is based on the so-called thermal vorticity [8]. To provide the comparison we calculated (see Fig. 6) the thermal vorticity field and respective polarization. While a 

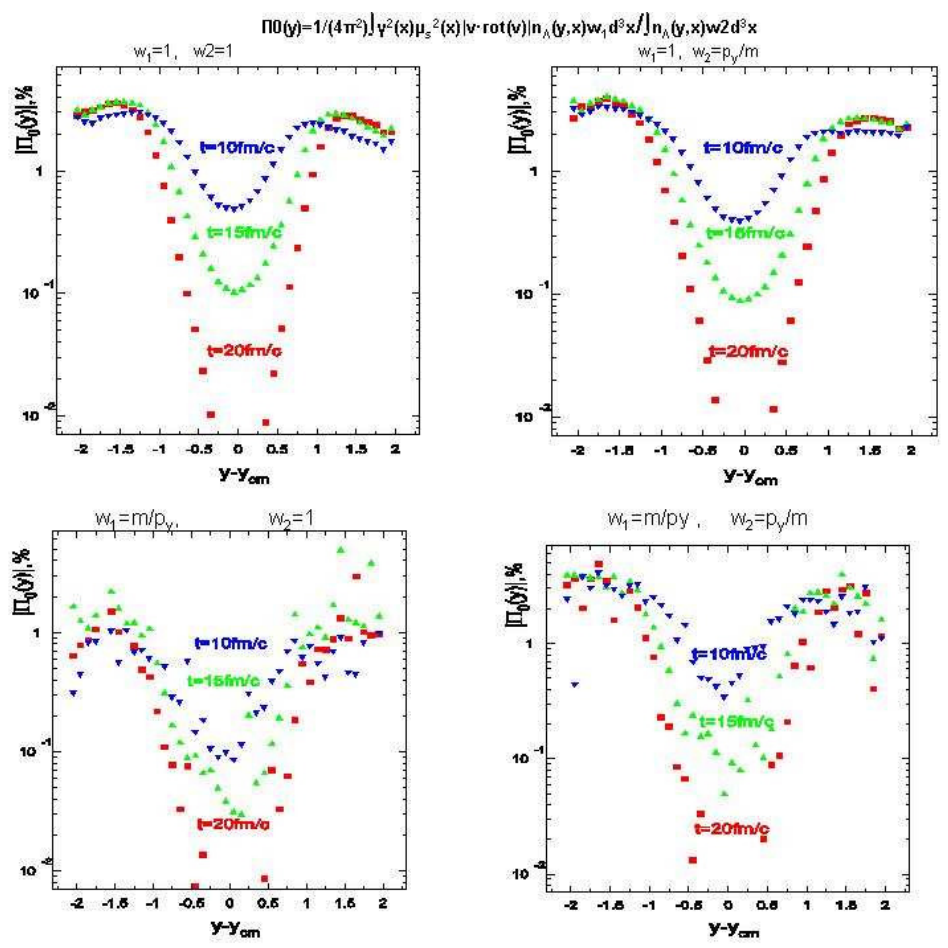

Figure 5: The rapidity dependence of polarization in helicity-based approach.

scale of the polarization in the thermal vorticity-based approach is several times larger, its rapidity dependence, surprisingly enough, appear to be similar in these rather distinct approaches.

\section{Conclusions and Outlook}

We investigated vorticity and hydrodynamical helicity in noncentral heavy-ion collisions in the framework of the kinetic quark-gluon string model. We have observed that the vorticity is predominantly localized in a relatively thin layer $(2 \div 3 \mathrm{fm})$ on the boundary between the participant and spectator nucleons. This might be qualitatively understood in the spirit of the core-corona type models $[18,19]$.

Thus, the gradients of the velocities in the region occupied by the participants are small due to the compensation of momenta between the target and projectile particles in the c.m. frame. As the result the vorticity is substantial only in the thin transition layer between the participant (i.e., core) and the spectator (i.e., corona) regions. We found the novel effect of the helicity separation in heavy-ion collisions when it has the different signs below and above of the reaction plane. We have investigated its dependence on the type of nuclei and collision energy and observed that it is maximal in the NICA energy range. We have also calculated the degree of alignment of the velocity and vorticity which is maximal for the Beltrami flows (manifesting elegant symmetry structures [21]) whose relativistic generalization is currently under investigation [22]. 

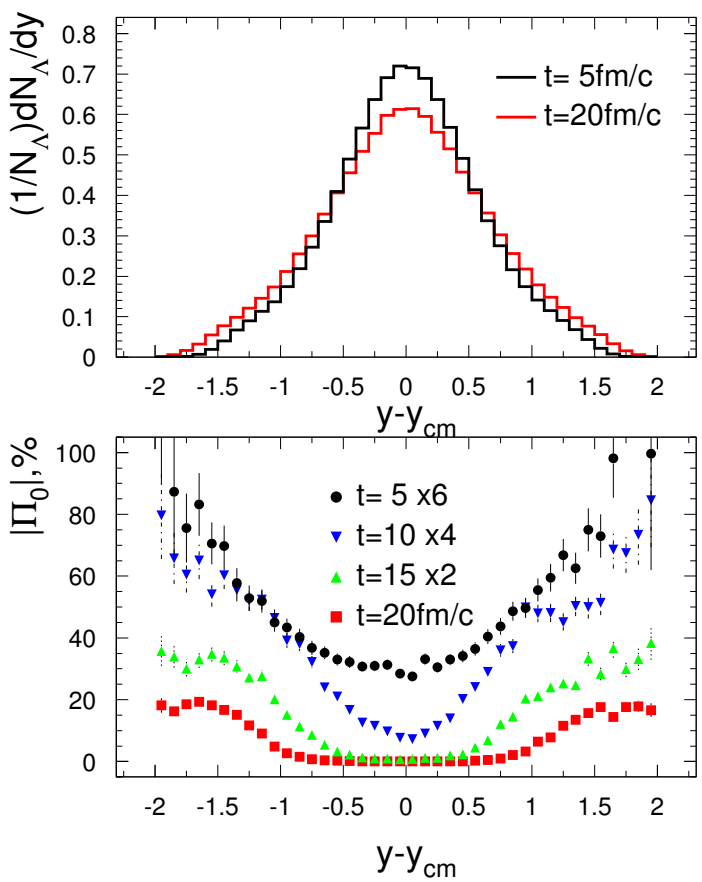

Figure 6: The rapidity dependence of the multiplicity and polarization in the thermal vorticity-based approach. The magnitudes of polarization at different times are rescaled by the identified factors.

We used the obtained values of helicity for estimates of $\Lambda$ hyperons polarization in heavy-ion collisions at NICA energy range due to earlier suggested [3] mechanism. The resulting polarization is bout $1 \%$ and may be studied experimentally. Of course, its more detailed theoretical investigations are required.

In particular, the discovery of extra $T^{2}$ term [23] raises again the question why polarization was not observed at RHIC. Here one may refer to the (exponential) dilution of polarization by temperature effects (similar to what happens at much larger scale in polarized targets) although this problem certainly requires further investigation.

\section{References}

[1] K. Fukushima, D.E. Kharzeev and H.J. Warringa, Phys. Rev. D 78, 074033 (2008)

[2] D. Kharzeev and A. Zhitnitsky, Nucl. Phys. A 797, 67 (2007) 
[3] O. Rogachevsky, A. Sorin and O. Teryaev, Phys. Rev. C 82, 054910 (2010) [arXiv:1006.1331 [hep-ph]].

[4] M. Baznat, K. Gudima, A. Sorin and O. Teryaev, Phys. Rev. C 88, 061901 (2013) [arXiv:1301.7003 [nucl-th]].

[5] O. Teryaev and R. Usubov, arXiv:1406.4451 [nucl-th].

[6] T. Kalaydzhyan, Phys. Rev. D 89, no. 10, 105012 (2014) [arXiv:1403.1256 [hep-th]].

[7] J. -H. Gao, Z. -T. Liang, S. Pu, Q. Wang and X. -N. Wang, Phys. Rev. Lett. 109, 232301 (2012)

[8] F. Becattini, L. Csernai and D. J. Wang, Phys. Rev. C 88, no. 3, 034905 (2013) [arXiv:1304.4427 [nucl-th]].

[9] M. I. Baznat, K. K. Gudima, A. S. Sorin and O. V. Teryaev, arXiv:1507.04652 [nucl-th].

[10] V.D. Toneev, K.K. Gudima, Nucl. Phys. A 400, 173c (1983)

[11] V.D. Toneev, N.S. Amelin, K.K. Gudima, S.Yu. Sivoklokov, Nucl. Phys. A 519, 463c (1990).

[12] N.S. Amelin, E.F. Staubo, L.S. Csernai et al., Phys.Rev. C 44, 1541 (1991).

[13] R.D. Field, R.P. Feynman, Nucl. Phys. B 136, 1 (1978).

[14] N.S. Amelin, K.K. Gudima, S.Yu. Sivoklokov, V.D. Toneev, Sov. J. Nucl. Phys. 52, 272 (1990).

[15] D.T. Son and P. Surowka, Phys. Rev. Lett. 103, 191601 (2009) [arXiv:0906.5044 [hep-th]].

[16] L. P. Csernai, D. D. Strottman and C. .Anderlik, Phys. Rev. C 85 (2012) 054901 [arXiv:1112.4287 [nucl-th]].

[17] J. Steinheimer, private communication.

[18] J. Aichelin and K. Werner, aXiv:0810.4465[nucl-th]

[19] J. Steinheimer and M. Bleicher, arXiv:1104.3981[nucl-th]

[20] X. Artru, M. Elchikh, J. -M. Richard, J. Soffer and O. V. Teryaev, Phys. Rept. 470, 1 (2009)

[21] P. Fre and A. S. Sorin, Phys. Part. Nucl. 46, no. 4, 497 (2015) [arXiv:1501.04604 [math-ph]].

[22] A.S. Sorin, O.V. Teryaev, work in progress.

[23] K. Landsteiner, E. Megias, L. Melgar and F. Pena-Benitez, JHEP 1109 (2011) 121 [arXiv:1107.0368 [hep-th]]. 